\title{
Renal impairment during pemetrexed maintenance in patients with advanced nonsmall cell lung cancer: a cohort study
}

\author{
Sabine Visser ${ }^{1,2,3}$, Jeannine Huisbrink ${ }^{4,5}$, Nils E. van 't Veer ${ }^{4}$, \\ Jermo J. van Toor ${ }^{2}$, Anton J.M. van Boxem6, Nico C. van Walree ${ }^{1}$, \\ Bruno H. Stricker ${ }^{3,7}$ and Joachim G.J.V. Aerts ${ }^{1,2}$
}

Affiliations: ${ }^{1}$ Dept of Pulmonary Medicine, Amphia Hospital, Breda, The Netherlands. ${ }^{2}$ Dept of Pulmonary Medicine, Erasmus MC Cancer Institute, Rotterdam, The Netherlands. ${ }^{3}$ Dept of Epidemiology, Erasmus MC University Medical Center, Rotterdam, The Netherlands. ${ }^{4}$ Dept of Clinical Pharmacy, Amphia Hospital, Breda, The Netherlands. ${ }^{5}$ Dept of Pharmacy, Franciscus Gasthuis \& Vlietland, Rotterdam, The Netherlands. ${ }^{6}$ Dept of Pulmonary Medicine, Bravis Hospital, Roosendaal, The Netherlands. ${ }^{7}$ Inspectorate of Health Care, Utrecht, The Netherlands.

Correspondence: Bruno H. Stricker, Dept of Epidemiology, Erasmus Medical Center, PO Box 2040, 3000 CA Rotterdam, The Netherlands. E-mail: b.strickerderasmusmc.nl

@ERSpublications

There is a significant risk of renal impairment during pemetrexed maintenance, which may jeopardise further treatment http://ow.ly/q8r830lnNZR

Cite this article as: Visser S, Huisbrink J, van 't Veer NE, et al. Renal impairment during pemetrexed maintenance in patients with advanced nonsmall cell lung cancer: a cohort study. Eur Respir J 2018; 52: 1800884 [https://doi.org/10.1183/13993003.00884-2018].

ABSTRACT Optimal survival benefit from different lines of anticancer treatment in advanced nonsmall cell lung cancer (NSCLC) requires conservation of renal function. We evaluated the development of renal impairment during pemetrexed maintenance.

In a prospective multicentre cohort study, we evaluated the incidence of acute/chronic kidney disease (AKD/CKD), its related treatment discontinuation frequency and associated clinical variables with $\mathrm{AKD}$ in patients with stage IIIB/IV NSCLC treated with pemetrexed maintenance. We validated the findings in an independent cohort.

190 patients received pemetrexed. In the primary cohort, 149 patients started induction, of whom 44 patients $(30 \%)$ continued maintenance. In the independent cohort, 41 patients received maintenance. During maintenance 13 patients $(30 \%)$ developed $\mathrm{AKD}$, leading to $\mathrm{CKD}$ and treatment discontinuation in eight patients $(62 \%)$ in the primary cohort. Higher estimated glomerular filtration rate (eGFR) (per unit $5 \mathrm{~mL} \cdot \mathrm{min}^{-1}$ per $1.73 \mathrm{~m}^{2}$ ) before maintenance and induction (OR 0.70, 95\% CI $0.54-0.90$ and OR $0.78,95 \%$ CI $0.62-0.98$, respectively) and relative decline (per 10\%) in eGFR during induction (OR 2.54, 95\% CI 1.36-4.74) were associated with AKD during maintenance. In the independent cohort, 20 patients (49\%) developed $\mathrm{AKD}$, leading to $\mathrm{CKD}$ in 11 patients $(55 \%)$ and treatment discontinuation in six patients $(30 \%)$.

Patients are at risk for renal impairment during pemetrexed maintenance, which may jeopardise further lines of anticancer treatment. 


\section{Introduction}

Pemetrexed is widely used as first- and second-line treatment in nonsquamous nonsmall cell lung cancer (NSCLC) without actionable driver mutations or high programmed death ligand 1 (PD-L1) expression [1]. More recently, first-line platinum-based treatment with pemetrexed combined with pembrolizumab prolonged overall survival compared with chemotherapy regardless of PD-L1 expression [2]. In patients without disease progression after platinum-based induction therapy, pemetrexed is recommended as maintenance treatment [3-6]. Both immunotherapy and the combination of docetaxel with antiangiogenic agents have demonstrated their superior efficacy compared with conventional chemotherapy, and have been approved for second-line treatment $[7,8]$. However, to gain optimal survival benefit from all these agents, patients should be able to start as well as continue multiple lines of treatment for which it is required to maintain an adequate renal function $[9,10]$.

Patients with (lung) cancer are at increased risk of developing acute kidney injury (AKI) [11]. In addition to exposure to nephrotoxic chemotherapeutic agents, decline in renal function in these patients is due to cancer- or chemotherapy-induced true or effective volume depletion, the advanced age of the patients and nephrotoxic concomitant medication [12]. The mechanism of renal injury by pemetrexed is postulated to be mainly tubulointerstitial, as pemetrexed enters the proximal tubular cells at the basolateral membrane by the reduced folate carrier and is transported through the folate receptor at its apical site. Once inside the tubular cells, pemetrexed undergoes polyglutamylation that results in intracellular retention and an increase in affinity towards enzymes involved in folate metabolism, leading to tubular injury due to impaired DNA synthesis [12,13]. Although pemetrexed administration is not recommended in patients with a creatinine clearance $<45 \mathrm{~mL} \cdot \mathrm{min}^{-1}$ per $1.73 \mathrm{~m}^{2}$ [14], studies have shown that even milder pre-existing renal impairment is a risk factor for drug-induced nephrotoxicity $[15,16]$.

Irrespective of its nature, AKI is a predictor of immediate and long-term unfavourable outcomes [17-19]. Moreover, AKI is an important risk factor for the development of chronic kidney disease (CKD) [20] and may jeopardise further cancer treatment [21]. Sustained impairment of the kidney function after discontinuation of pemetrexed maintenance therapy has been described in several case reports [22, 23]. The PARAMOUNT study (a study of induction and maintenance treatment of advanced nonsquamous NSCLC) reported renal impairment in $<10 \%$ of the patients treated with pemetrexed maintenance and $<5 \%$ of the patients discontinued treatment due to renal toxicity [24]. However, this trial population was highly selected and might underestimate the risk and consequences of renal toxicity in daily clinical practice.

Therefore, our objective was to describe the development of acute and chronic renal impairment during maintenance treatment with pemetrexed, and its impact on treatment decisions in a real-world setting.

\section{Materials and methods}

Prospective multicentre cohort (primary cohort)

The PERSONAL (PEmetrexed and biomaRkerS: an observatiONAL) study is a prospective multicentre cohort study of adult patients with locally advanced or metastatic (stage IIIB/IV) nonsquamous NSCLC and unresectable mesothelioma receiving platinum-combined pemetrexed as first-line treatment and pemetrexed monotherapy as second-line treatment. Patients were recruited between October 2012 and November 2014 from a university hospital (Erasmus University Medical Center, Rotterdam), two large teaching hospitals specialised in lung cancer care (Amphia Hospital, Breda and Franciscus Gasthuis \& Vlietland, Rotterdam) and a regional hospital (Bravis Hospital, Roosendaal) in the Netherlands. Patients who received pemetrexed as second-line treatment and patients with unresectable mesothelioma were excluded from analyses in the present study. The PERSONAL cohort will be denoted as the "primary cohort" in this article. All patients provided written informed consent. The study was approved by the Institutional Review Board of the Erasmus University Medical Center.

Per standard of care, platinum-combined pemetrexed chemotherapy was administered as an intravenous infusion every 3 weeks for a maximum of 4 cycles. The administered dosages of pemetrexed and cisplatin were calculated according to the body surface area $\left(500\right.$ and $75 \mathrm{mg} \cdot \mathrm{m}^{-2}$, respectively) [14]. Carboplatin dosage was calculated based on estimated glomerular filtration rate (eGFR) and the target area under the curve values of 5 or 6 following the Calvert formula [25]. If the chemotherapy schedule involved cisplatin, pre- and post-hydration treatment was given per protocol. Patients were recommended to continue with pemetrexed maintenance therapy if they had no progressive disease, no intolerable toxicities and underwent no sequential radiotherapy or surgery.

Baseline serum creatinine $\left(\mu \mathrm{mol} \cdot \mathrm{L}^{-1}\right)$ was obtained prior to the initial chemotherapy cycle. Subsequently, serum creatinine was measured prior to and weekly after each chemotherapy administration during the induction therapy. During maintenance treatment blood samples were only extracted prior to pemetrexed 
administration and at day 14 of each cycle. Estimations of renal function were made by calculation of the eGFR ( $\mathrm{mL} \cdot \mathrm{min}^{-1}$ per $1.73 \mathrm{~m}^{2}$ ) using the Chronic Kidney Disease Epidemiology Collaboration equation [26]. Renal adverse events were registered according to the Common Terminology Criteria of Adverse Events (CTCAE) version 3.0, for comparison with the registration trial of pemetrexed maintenance [24], and the updated version 4.03 (table 1).

\section{Independent cohort}

To validate findings in the primary cohort, we selected all patients with advanced NSCLC who started treatment with pemetrexed maintenance between November 2014 and December 2016 in one hospital (Amphia Hospital). We used the pharmacy database of this centre to construct a second independent cohort of patients who received pemetrexed maintenance treatment after patient enrolment in the PERSONAL study had finished. Prior to maintenance treatment, these patients received first-line platinum-combined induction treatment with pemetrexed and hydration per standard of care (see previous section) creatinine level before start of induction and maintenance, and during maintenance prior to each pemetrexed administration. As data in this cohort were collected retrospectively, no approval by a medical research and ethics committee was necessary according to Dutch guidelines.

\section{Definitions of acute and chronic kidney disease}

In both cohorts, patients with acute kidney disease (AKD) and CKD during induction and maintenance therapy were identified in accordance with the Kidney Disease: Improving Global Outcomes (KDIGO) clinical practice guidelines (table 2) [27, 28].

In addition to the development of $\mathrm{CKD}$, we registered clinical consequences related to decreased renal function in terms of discontinuation of therapy, hospitalisation, and dose adjustments and postponements.

\section{Statistical analysis}

Sociodemographic and clinical variables were described for all patients who were included in both cohorts. Patients from the primary cohort who underwent maintenance treatment were categorised into two groups (eGFR $<90$ versus $\geqslant 90 \mathrm{~mL} \cdot \mathrm{min}^{-1}$ per $1.73 \mathrm{~m}^{2}$ ) according to their renal function at baseline (start of induction treatment) and at the start of maintenance therapy. For these groups, we reported the percentages of patients with $\mathrm{AKD}, \mathrm{CKD}$ and clinical consequences with $95 \%$ confidence intervals calculated using the Wilson score method. The difference in incidence of AKD between these groups was examined using the Chi-squared or Fisher's exact test. With the use of logistic regression, we determined the univariate association of renal function before induction and maintenance (per unit eGFR of

\section{TABLE 1 Renal adverse events according to the Common Terminology Criteria of Adverse} Events (CTCAE)AQcon

\begin{tabular}{lc} 
Version & All-grades toxicity \\
\hline CTCAE 3.0 & $\begin{array}{c}\text { Creatinine above upper limit of normal } \\
\text { eGFR }<75 \% \text { lower limit of normal }\end{array}$ \\
CTCAE 4.03 & Acute kidney injury: creatinine level increase of $>26.5 \mu \mathrm{mol} \cdot \mathrm{L}^{-1}\left(>0.3 \mathrm{mg} \cdot \mathrm{dL}^{-1}\right)$ \\
Creatinine $>1.5$ times above baseline
\end{tabular}

eGFR: estimated glomerular filtration rate.

TABLE 2 Kidney Disease: Improving Global Outcomes clinical practice guidelines for identification of acute kidney disease (AKD) and chronic kidney disease (CKD)

AKD

$$
\begin{gathered}
\text { eGFR }<60 \mathrm{~mL} \cdot \mathrm{min}^{-1} \text { per } 1.73 \mathrm{~m}^{2} \text { for }<3 \text { months } \\
\text { or } \\
\text { Decrease in eGFR by }>35 \% \\
\text { or } \\
\text { Increase in serum creatinine }>50 \% \text { for }<3 \text { months } \\
\text { eGFR }<60 \mathrm{~mL} \cdot \mathrm{min}^{-1} \text { per } 1.73 \mathrm{~m}^{2} \text { for }>3 \text { months }
\end{gathered}
$$

eGFR: estimated glomerular filtration rate. ${ }^{\#}$ : in patients with a baseline eGFR $<60 \mathrm{~mL} \cdot \mathrm{min}^{-1}$ per $1.73 \mathrm{~m}^{2}$ only changes in eGFR and serum creatinine during the next 3 months were used as criteria for AKD. 
$5 \mathrm{~mL} \cdot \mathrm{min}^{-1}$ per $1.73 \mathrm{~m}^{2}$ ), change of renal function during induction (relative to baseline per 10\%), and other patient- and treatment-related factors with the incidence of AKD during maintenance. To verify findings from our prospective cohort study, we repeated these analyses in the second independent cohort.

All statistical analyses were performed using SPSS version 22.0 (IBM, Armonk, NY, USA). p $<0.05$ was considered as statistically significant.

\section{Results}

In total, 190 patients who received treatment with pemetrexed were included in the current study. In the primary cohort, 149 patients with advanced NSCLC who started first-line induction treatment with pemetrexed were enrolled. Of these, 44 patients (29.5\%) ultimately received one or more cycles of pemetrexed maintenance treatment (figure 1). The second independent cohort consisted of 41 patients with advanced NSCLC who had received one or more cycles cycle of pemetrexed maintenance therapy after first-line induction treatment.

All patient and treatment characteristics of patients in both cohorts are outlined in table 3 . In the primary cohort, a higher percentage of patients with maintenance pemetrexed $(n=44)$ had metastatic disease $(p=0.003)$ and they had higher serum albumin $(p=0.001)$ than patients who only received induction treatment $(n=105)$. Between patients who underwent maintenance in both cohorts, there were no significant differences and platinum combination treatments were similar. Slightly more female patients underwent pemetrexed maintenance in the second independent cohort than in the primary cohort $(65.9 \%$ versus $50.0 \% ; \mathrm{p}=0.188)$. Median (interquartile range (IQR)) follow-up time was $3.2(1.9-6.1)$ months in the second independent cohort and $3.5(1.4-8.3)$ months in the primary cohort.

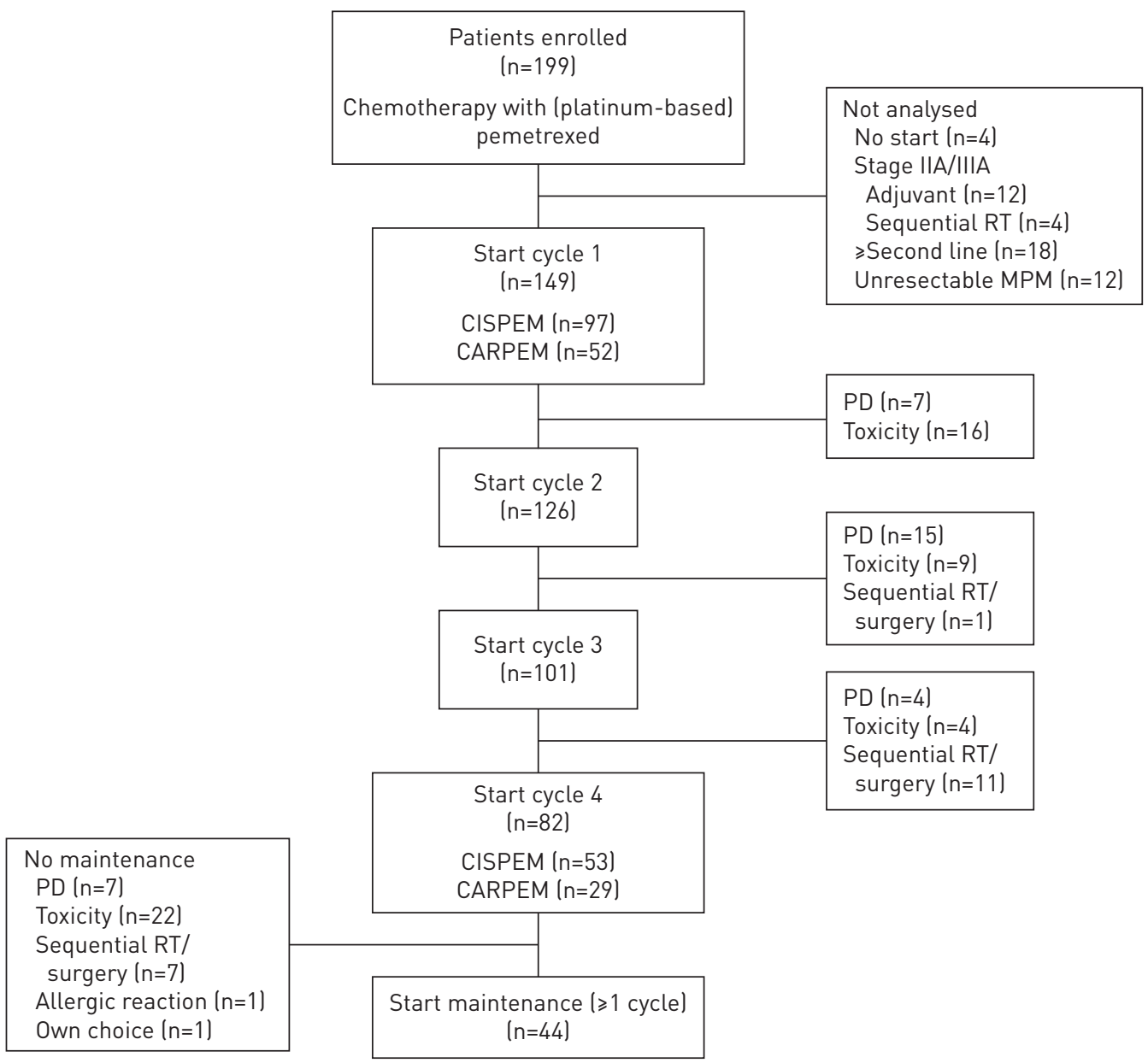

FIGURE 1 Flowchart of patients in the primary cohort. RT: radiotherapy; MPM: malignant pleural mesothelioma; CISPEM: combined cisplatin and pemetrexed; CARPEM: combined carboplatin and pemetrexed; PD: progressive disease. 
TABLE 3 Characteristics of all 190 patients with advanced nonsmall cell lung cancer who received treatment with pemetrexed

\begin{tabular}{|c|c|c|c|}
\hline & \multicolumn{2}{|c|}{ Primary cohort } & \multirow{2}{*}{$\begin{array}{c}\text { Independent } \\
\text { cohort }\end{array}$} \\
\hline & $\begin{array}{c}\text { No maintenance } \\
\text { pemetrexed }\end{array}$ & $\begin{array}{l}\text { Maintenance } \\
\text { pemetrexed }\end{array}$ & \\
\hline Patients & 105 & 44 & 41 \\
\hline Age years & $63.7 \pm 9.4$ & $62.9 \pm 7.5$ & $62.8 \pm 6.7$ \\
\hline Male & 52 (49.5) & $22(50.0)$ & $14(34.1)$ \\
\hline \multicolumn{4}{|l|}{ Ethnicity } \\
\hline Caucasian & 100 (95.2) & $42(95.5)$ & 38 (92.7) \\
\hline Black & $1(1.0)$ & 0 & 0 \\
\hline Asian & $2(1.9)$ & 0 & 0 \\
\hline Other & $2(1.9)$ & $2(4.5)$ & $3(7.3)$ \\
\hline BMI $\mathrm{kg} \cdot \mathrm{m}^{-2}$ & $24.3 \pm 3.9$ & $25.3 \pm 3.7$ & $24.8 \pm 5.3$ \\
\hline Pack-years & $38.3 \pm 36.4$ & $34.7 \pm 23.3$ & $34.0 \pm 21.0$ \\
\hline \multicolumn{4}{|l|}{ Type of tumour } \\
\hline Adenocarcinoma & $102(97.1)$ & $44(100)$ & $41(100)$ \\
\hline Large cell carcinoma & $3(2.9)$ & 0 & 0 \\
\hline \multicolumn{4}{|l|}{ Cancer stage } \\
\hline Locally advanced (IIIA) & 0 & 0 & $1(2.4)^{\#}$ \\
\hline Locally advanced (IIIB) & 20 (19.0) & 0 & $1(2.4)$ \\
\hline Metastatic (IV) & $85(81.0)$ & $44(100)$ & $39(95.1)$ \\
\hline \multicolumn{4}{|l|}{ Line of induction treatment } \\
\hline First line & 105 (100) & $44(100)$ & $41(100)$ \\
\hline \multicolumn{4}{|l|}{ Platinum combination } \\
\hline Cisplatin & 65 (61.9) & $32(72.7)$ & $31(75.6)$ \\
\hline Carboplatin & $40(38.1)$ & $12(27.3)$ & $13(24.4)$ \\
\hline \multicolumn{4}{|l|}{ Laboratory values } \\
\hline Creatinine $\mathrm{mL} \cdot \mathrm{min}^{-1}$ & $61.0(49.0-72.5)$ & $57.5(52.0-70.0)$ & $64.0(51.5-79.0)$ \\
\hline eGFR $\mathrm{mL} \cdot \mathrm{min}^{-1}$ per $1.73 \mathrm{~m}^{2}$ & $96.9(85.4-104.7)$ & $97.6(88.6-106.1)$ & $95.4(79.4-101.0)$ \\
\hline eGFR $<60 \mathrm{~mL} \cdot \mathrm{min}^{-1}$ per $1.73 \mathrm{~m}^{2}$ & $6(5.7)$ & $2(4.5)$ & $1(2.4)$ \\
\hline Albumin $\mathrm{g} \cdot \mathrm{L}^{-1}$ & $38.6 \pm 5.3$ & $41.3 \pm 3.8$ & Unknown \\
\hline \multicolumn{4}{|l|}{ Comorbidity } \\
\hline Cardiovascular disease & $47(44.8)$ & $16(36.4)$ & $18(43.9)$ \\
\hline Diabetes mellitus & $17(16.2)$ & $4(9.1)$ & $5(12.2)$ \\
\hline
\end{tabular}

Data are presented as $\mathrm{n}$, mean $\pm \mathrm{SD}, \mathrm{n}(\%)$ or median (interquartile range). BMI: body mass index; eGFR: estimated glomerular filtration rate. " : patient received only palliative chemotherapy (lymphangitis carcinomatosa).

Renal impairment in the primary cohort

Induction treatment

Calculated eGFR values at baseline were significantly different between the patients treated with combined cisplatin and pemetrexed (CISPEM) and with combined carboplatin and pemetrexed (CARPEM) (98.1 \pm 16.0 versus $88.7 \pm 15.9 \mathrm{~mL} \cdot \mathrm{min}^{-1}$ per $1.73 \mathrm{~m}^{2}$; $\left.\mathrm{p}=0.001\right)$. Over the total induction treatment of 4 cycles, the mean eGFR decreased in patients treated with CISPEM $(n=53)$ in contrast to the mean eGFR in patients treated with CARPEM $(\mathrm{n}=29)\left(-9.1 \pm 9.5\right.$ versus $-2.0 \pm 11.0 \mathrm{~mL} \cdot \mathrm{min}^{-1}$ per $\left.1.73 \mathrm{~m}^{2} ; \mathrm{p}=0.003\right)$. Weekly median eGFR values of patients receiving combined platinum induction treatment are described in the supplementary material.

\section{Maintenance}

The median (IQR) number of maintenance pemetrexed cycles was 5 (2-12) and the eGFR before administration of the first maintenance cycle was $86.3(71.6-97.2) \mathrm{mL} \cdot \mathrm{min}^{-1}$ per $1.73 \mathrm{~m}^{2}$. During maintenance treatment with pemetrexed 13 out of the 44 patients $(29.5 \%)$ developed AKD according to KDIGO definitions. From these 13 patients, 10 patients (77\%) had all-grades renal adverse events according to CTCAE 4.03 compared with only seven patients (54\%) using CTCAE 3.0. Hence, using CTCAE 3.0 we found only $16 \%$ of patients experienced renal adverse events.

Individual courses of patients' renal function are shown in figure 2. Compared with patients with eGFR $\geqslant 90 \mathrm{~mL} \cdot \mathrm{min}^{-1}$ per $1.73 \mathrm{~m}^{2}$ at the start of maintenance, patients with a mildly decreased renal function (eGFR $<90 \mathrm{~mL} \cdot \mathrm{min}^{-1}$ per $1.73 \mathrm{~m}^{2}$ ) more frequently developed AKD (11 out of 23 versus two out of 21; 

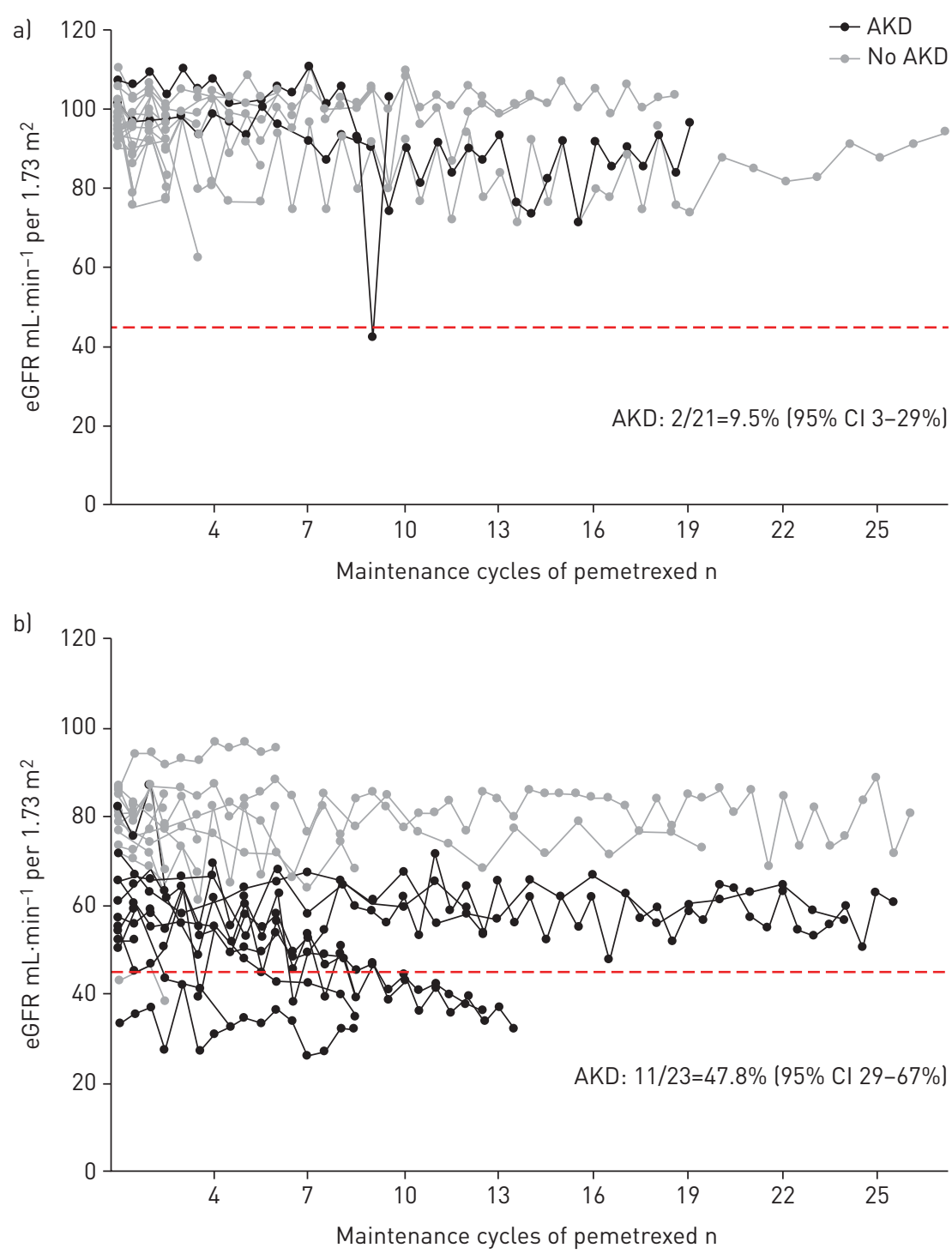

FIGURE 2 Renal function and development of acute kidney disease (AKD) during pemetrexed maintenance therapy in the primary cohort $(\mathrm{n}=44)$. eGFR: estimated glomerular filtration rate. a) eGFR $\geqslant 90 \mathrm{~mL} \cdot \mathrm{min}^{-1}$ per $1.73 \mathrm{~m}^{2}(\mathrm{n}=21)$ and b) eGFR $<90 \mathrm{~mL} \cdot \mathrm{min}^{-1}$ per $1.73 \mathrm{~m}^{2}(\mathrm{n}=23)$. Data are presented as individual measurements of renal function together with course of renal function during maintenance therapy of individuals who developed AKD and did not develop AKD. The dashed red line indicates eGFR=45 $\mathrm{mL} \cdot \mathrm{min}^{-1}$ per $1.73 \mathrm{~m}^{2}$, the value below which pemetrexed administration is not recommended.

$\mathrm{p}=0.005$ ) and their renal function more often decreased below the recommendation threshold of pemetrexed administration (eGFR $<45 \mathrm{~mL} \cdot \mathrm{min}^{-1}$ per $1.73 \mathrm{~m}^{2}$, six out of 21 versus one out of 21; $\mathrm{p}=0.017$ ). Two patients with $\mathrm{eGFR}<45 \mathrm{~mL} \cdot \mathrm{min}^{-1}$ per $1.73 \mathrm{~m}^{2}$ already before maintenance were excluded from this analysis.

Per unit $5 \mathrm{~mL} \cdot \mathrm{min}^{-1}$ per $1.73 \mathrm{~m}^{2}$, higher eGFR values both before maintenance and before induction treatment were associated with a lower risk of AKD (OR 0.70, 95\% CI 0.54-0.90 and OR 0.78, 95\% CI $0.62-0.98$, respectively) (table 4). In contrast, a $10 \%$ decline in eGFR during induction relative to baseline was associated with an increased probability of AKD (OR 2.54, 95\% CI 1.36-4.74). In patients with $\mathrm{AKD}$ the mean $\pm \mathrm{SD}$ decrease of eGFR during induction was $-12.2 \pm 8.9 \mathrm{~mL} \cdot \mathrm{min}^{-1}$ per $1.73 \mathrm{~m}^{2}$ compared with $-2.1 \pm 8.4 \mathrm{~mL} \cdot \mathrm{min}^{-1}$ per $1.73 \mathrm{~m}^{2}$ in patients without AKD.

\section{Clinical implications}

The development of CKD and clinical consequences of renal impairment during maintenance therapy are outlined in figure 3. Of the 13 patients $(30 \%)$ who developed AKD during maintenance therapy, eight patients $(62 \%)$ ultimately developed CKD. Eight of the 13 patients with AKD (62\%) were forced to 
TABLE 4 Univariate analysis of clinical and treatment-related factors associated with acute kidney disease during pemetrexed maintenance

\begin{tabular}{|c|c|c|c|c|}
\hline & \multicolumn{2}{|c|}{ Primary cohort $\#$} & \multicolumn{2}{|c|}{ Independent cohort ${ }^{\Uparrow}$} \\
\hline & OR $(95 \% \mathrm{CI})$ & p-value & OR $(95 \% \mathrm{CI})$ & p-value \\
\hline Age & $1.07(0.98-1.18)$ & 0.14 & $0.98(0.89-1.07)$ & 0.62 \\
\hline Sex: male versus female & $0.52(0.14-1.93)$ & 0.34 & $0.28(0.068-1.11)$ & 0.069 \\
\hline $\begin{array}{l}\text { History of cardiovascular disease: } \\
\text { yes versus no }\end{array}$ & $0.70(0.18-2.80)$ & 0.62 & $0.73(0.21-2.53)$ & 0.62 \\
\hline CISPEM during induction: yes versus no & $2.62(0.49-14.11)$ & 0.26 & $0.94(0.23-3.90)$ & 0.93 \\
\hline Number of cycles pemetrexed maintenance & $1.08(1.0-1.17)$ & 0.059 & $1.09(0.96-1.23)$ & 0.2 \\
\hline eGFR decrease during induction ${ }^{+}$ & $2.54(1.36-4.74)$ & 0.004 & $1.56(1.03-2.36)$ & 0.038 \\
\hline eGFR before induction ${ }^{\S}$ & $0.78(0.62-0.98)$ & 0.032 & $0.78(0.62-0.98)$ & 0.035 \\
\hline eGFR before maintenance ${ }^{\S}$ & $0.70(0.54-0.90)$ & 0.005 & $0.64(0.48-0.84)$ & 0.001 \\
\hline \multicolumn{5}{|c|}{$\begin{array}{l}\text { CISPEM: combined cisplatin and pemetrexed; eGFR: estimated glomerular filtration rate. }{ }^{\#}: \mathrm{n}=44 ;{ }^{\uparrow}: \mathrm{n}=41 \text {; } \\
{ }^{+} \text {: eGFR change relative to baseline per } 10 \% \text {; }{ }^{\S} \text { : eGFR per unit } 5 \mathrm{~mL} \min ^{-1} \text { per } 1.73 \mathrm{~m}^{2} \text {. }\end{array}$} \\
\hline
\end{tabular}

discontinue maintenance treatment due to renal impairment. Importantly, all of these patients who developed CKD and stopped treatment already had mildly impaired renal function $\left(<90 \mathrm{~mL} \cdot \mathrm{min}^{-1}\right.$ per $1.73 \mathrm{~m}^{2}$ ) before the start of maintenance. Moreover, in patients whose renal function was already mildly impaired before induction $\left(<90 \mathrm{~mL} \cdot \mathrm{min}^{-1}\right.$ per $\left.1.73 \mathrm{~m}^{2}\right)$ the proportion of patients who had to discontinue treatment was higher than in patients with a normal eGFR (six out of 11 versus two out of 33; $\mathrm{p}=0.001$ ). Accordingly, patients more often developed AKD (six out of 11 versus seven out of 33; $\mathrm{p}=0.057$ ) and CKD (five out of 11 versus three out of 33; $\mathrm{p}=0.016$ ) if renal function was mildly impaired before induction.

\section{Renal impairment in the second independent cohort}

In the second independent cohort, the median (IQR) number of maintenance pemetrexed cycles was 4 (3-8) and the eGFR before administration of the first maintenance cycle was $80.6(63.4-93.3) \mathrm{mL} \cdot \mathrm{min}^{-1}$ per $1.73 \mathrm{~m}^{2} .20$ patients (49\%) developed AKD, of whom 11 patients (55\%) eventually developed CKD and six patients (30\%) discontinued pemetrexed maintenance. Similar to the primary cohort, all patients who developed CKD and stopped maintenance treatment had eGFR $<90 \mathrm{~mL} \cdot \mathrm{min}^{-1}$ per $1.73 \mathrm{~m}^{2}$ before start of maintenance.

We tested the same patient- and treatment-related variables for their relation with the development of AKD during maintenance as in the primary cohort (table 4). Again, per unit $5 \mathrm{~mL} \cdot \mathrm{min}^{-1}$ per $1.73 \mathrm{~m}^{2}$, higher eGFR values before maintenance and before induction treatment were univariably associated with a

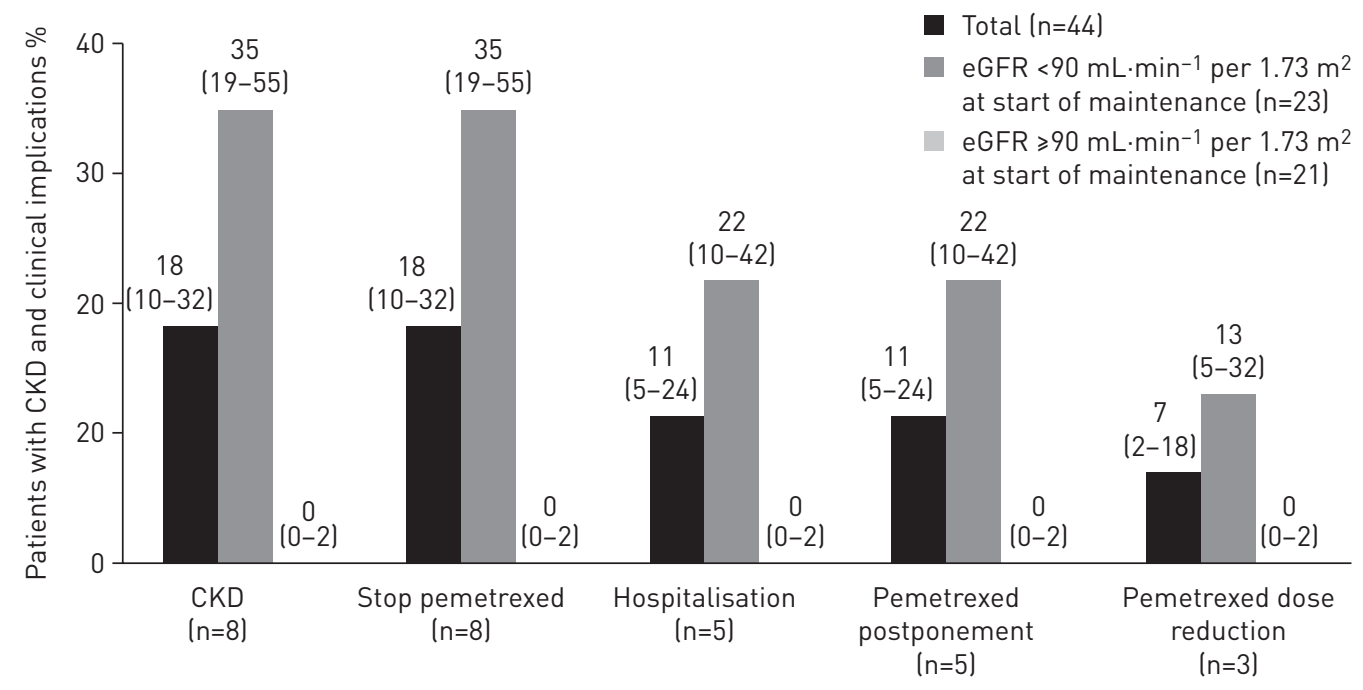

FIGURE 3 Chronic kidney disease (CKD) and clinical implications due to renal impairment during pemetrexed maintenance therapy in the primary cohort. eGFR: estimated glomerular filtration rate. Data are presented as $\%(95 \% \mathrm{CI})$. 
lower probability of AKD during maintenance (OR 0.64, 95\% CI $0.48-0.84$ and OR 0.78, 95\% CI 0.62 0.98 , respectively). Also, a $10 \%$ decline in eGFR during induction compared with baseline was related to an increased risk of AKD (OR 1.56, 95\% CI 1.03-2.36).

\section{Discussion}

In an era of accelerated development and adaptation of new agents with survival benefits for patients with advanced NSCLC, it becomes increasingly important to ascertain patients who are able to start and continue multiple lines of treatment. Our study shows serious concerns with regard to the preservation of an adequate renal function during pemetrexed maintenance therapy, which might expose patients to a suboptimal oncological treatment. In a real-world setting, one-third of patients with metastatic NSCLC developed AKD during pemetrexed maintenance therapy and half of these patients were forced to discontinue maintenance treatment. Moreover, in the majority of patients with AKD renal function did not recover (or only partially recovered) and these patients developed CKD. Importantly, these results were verified in an independent cohort of patients with advanced NSCLC treated with pemetrexed maintenance.

Approximately 20\% lower risk of occurrence of AKD during pemetrexed maintenance therapy was observed in patients per $5 \mathrm{~mL} \cdot \mathrm{min}^{-1}$ per $1.73 \mathrm{~m}^{2}$ higher eGFR before the start of induction therapy. The proportions of patients who developed $\mathrm{AKD}, \mathrm{CKD}$ and who discontinued maintenance treatment were significantly higher in patients with impaired renal function (eGFR $<90 \mathrm{~mL} \cdot \mathrm{min}^{-1}$ per $1.73 \mathrm{~m}^{2}$ ) at the start of maintenance and before induction. It has already been recognised that decreased renal function, even mildly, can predispose to chemotherapy-induced nephrotoxicity [15, 29]. SASSIER et al. [30] also reported a linkage between renal impairment before maintenance treatment and the higher probability of discontinuing double maintenance therapy with pemetrexed and bevacizumab. In contrast to our study, they did not find an association between renal function before induction and treatment discontinuation. In addition to almost $20 \%$ missing data of renal function before induction and a lack of patients with eGFR $<60 \mathrm{~mL} \cdot \mathrm{min}^{-1}$ per $1.73 \mathrm{~m}^{2}$ at baseline in that study, the different pathophysiology leading to renal damage due to bevacizumab might explain this difference.

During pemetrexed maintenance, patients were at $~ 2$-fold higher risk of developing AKD per 10\% decline of eGFR during induction therapy relative to baseline. Patients treated with CISPEM showed a decline in eGFR of $\sim 10 \mathrm{~mL} \cdot \mathrm{min}^{-1}$ per $1.73 \mathrm{~m}^{2}$, which is comparable to recent findings in patients who received cisplatin for treatment across multiple tumour types [13]. As treatment with CISPEM during induction therapy was not associated with AKD throughout the maintenance period, it is unlikely that nephrotoxicity during maintenance is solely a delayed cisplatin effect. This is supported by findings of follow-up studies in patients with various cancer types, including lung cancer, which demonstrated that declines in eGFR did not deteriorate after discontinuation of cisplatin [13,31]. Although not statistically significant $(p=0.06), A K D$ occurred more often in patients who received a higher number of pemetrexed maintenance cycles. A cumulative systemic dose of pemetrexed might play a role in the development of nephrotoxicity in these patients, as also recently suggested by LANGER et al. [32].

The nephrotoxic potential of pemetrexed has been described previously in clinical studies. In the pivotal PARAMOUNT trial [3], PUJOL et al. [24] reported all-grades renal toxicities according to CTCAE 3.0 in $7.8 \%$ of patients and treatment discontinuation in $4.5 \%$ of patients due to renal impairment during pemetrexed maintenance. Acknowledging small patient numbers, our study notes probable underestimation of renal toxicity by using CTCAE 3.0 compared with AKD (KDIGO). By taking into account absolute increases of creatinine and its relative increase from baseline, the results of the updated CTCAE 4.03 corresponded better with the AKD results. Additionally, the patient population in the PARAMOUNT trial was highly selected with regard to Eastern Cooperative Oncology Group performance status, renal function at baseline and concomitant medication, as opposed to our real-life population. Therefore, that trial probably underestimates the risk of renal insufficiency in daily practice. Although pemetrexed maintenance was combined with bevacizumab and therefore results cannot solely be attributed to pemetrexed, SASSIER et al. [30] reported renal adverse events resulting in treatment discontinuation in $17 \%$ of patients.

Due to a low event-rate number per subgroup in the primary and independent cohort, we could not perform a multivariate analysis to identify patient- and treatment-related variables associated with the development of $\mathrm{AKD}$ during maintenance therapy. As both cohorts differed with regard to frequency and timing of data collection of renal function by design, we did not consider it suitable to perform a combined analysis of these cohorts. We cannot exclude effect modification by the platinum compound, as all patients received CISPEM or CARPEM during induction treatment without a pemetrexed monotherapy comparator arm.

In conclusion, the results of this study in a real-life setting demonstrate that patients with advanced NSCLC are at risk to develop renal impairment during pemetrexed maintenance therapy. This has important clinical consequences, as the majority of these patients develop CKD, $\sim 15-20 \%$ are forced to 
stop maintenance treatment and further anticancer treatment may be jeopardised. Increased awareness and further exploration of renal protective strategies for patients at increased risk might be beneficial, such as continuation of hydration during pemetrexed maintenance.

Acknowledgements: We thank Hans in 't Veen (Franciscus Gasthuis \& Vlietland, Rotterdam, The Netherlands) for providing data.

Conflict of interest: S. Visser reports grants from ZonMw (grant number 152001017), during the conduct of the study. J. Huisbrink has nothing to disclose. N.E. van 't Veer has nothing to disclose. J.J. van Toor has nothing to disclose. A.J. M. van Boxem has nothing to disclose. N.C. van Walree has nothing to disclose. B.H. Stricker reports grants from ZonMw (grant number 152001017), during the conduct of the study. J.G.J.V. Aerts reports grants from ZonMw (grant number 152001017), during the conduct of the study; and has consultant/advisory roles with Eli Lilly and Company, Roche, Bristol-Myers Squibb, MSD and Boehringer Ingelheim, outside the submitted work.

Support statement: This work was supported by the Netherlands Organisation for Health Research and Development (ZonMw) (grant number 152001017). Funding information for this article has been deposited with the Crossref Funder Registry.

\section{References}

1 Hanna N, Johnson D, Temin S, et al. Systemic therapy for stage IV non-small-cell lung cancer: American Society of Clinical Oncology clinical practice guideline update. J Clin Oncol 2017; 35: 3484-3515.

2 Gandhi L, Rodríguez-Abreu D, Gadgeel S, et al. Pembrolizumab plus chemotherapy in metastatic non-small-cell lung cancer. N Engl J Med 2018; 378: 2078-2092.

3 Paz-Ares L, De Marinis F, Dediu M, et al. Maintenance therapy with pemetrexed plus best supportive care versus placebo plus best supportive care after induction therapy with pemetrexed plus cisplatin for advanced non-squamous non-small-cell lung cancer (PARAMOUNT): a double-blind, phase 3, randomised controlled trial. Lancet Oncol 2012; 13: 247-255.

4 Ciuleanu T, Brodowicz T, Zielinski C, et al. Maintenance pemetrexed plus best supportive care versus placebo plus best supportive care for non-small-cell lung cancer: a randomised, double-blind, phase 3 study. Lancet 2009; 374: $1432-1440$.

5 Herbst RS, Baas P, Kim D-W, et al. Pembrolizumab versus docetaxel for previously treated, PD-L1-positive, advanced non-small-cell lung cancer (KEYNOTE-010): a randomised controlled trial. Lancet 2016; 387: $1540-1550$.

6 Seto T, Kato T, Nishio M, et al. Erlotinib alone or with bevacizumab as first-line therapy in patients with advanced non-squamous non-small-cell lung cancer harbouring EGFR mutations (JO25567): an open-label, randomised, multicentre, phase 2 study. Lancet Oncol 2014; 15: 1236-1244.

7 Borghaei H, Paz-Ares L, Horn L, et al. Nivolumab versus docetaxel in advanced nonsquamous non-small-cell lung cancer. N Engl J Med 2015; 373: 1627-1639.

8 Reck M, Kaiser R, Mellemgaard A, et al. Docetaxel plus nintedanib versus docetaxel plus placebo in patients with previously treated non-small-cell lung cancer (LUME-Lung 1): a phase 3, double-blind, randomised controlled trial. Lancet Oncol 2014; 15: 143-155.

9 Sacher AG, Le LW, Lau A, et al. Real-world chemotherapy treatment patterns in metastatic non-small cell lung cancer: are patients undertreated? Cancer 2015; 121: 2562-2569.

10 Soon YY, Stockler MR, Askie LM, et al. Duration of chemotherapy for advanced non-small-cell lung cancer: a systematic review and meta-analysis of randomized trials. J Clin Oncol 2009; 27: 3277-3283.

11 Christiansen CF, Johansen MB, Langeberg WJ, et al. Incidence of acute kidney injury in cancer patients: a Danish population-based cohort study. Eur J Intern Med 2011; 22: 399-406.

12 Perazella MA. Onco-nephrology: renal toxicities of chemotherapeutic agents. Clin J Am Soc Nephrol 2012; 7: 1713-1721.

13 Latcha S, Jaimes EA, Patil S, et al. Long-term renal outcomes after cisplatin treatment. Clin J Am Soc Nephrol 2016; 11: 1173-1179.

14 Eli Lilly. ALIMTA (pemetrexed disodium): prescribing information. 2008. www.accessdata.fda.gov/drugsatfda docs/label/2009/021462s021lbl.pdf Date last accessed: February 2, 2018.

15 Ha SH, Park JH, Jang HR, et al. Increased risk of everolimus-associated acute kidney injury in cancer patients with impaired kidney function. BMC Cancer 2014; 14: 906.

16 Clos S, Rauchhaus P, Severn A, et al. Long-term effect of lithium maintenance therapy on estimated glomerular filtration rate in patients with affective disorders: a population-based cohort study. Lancet Psychiatry 2015; 2: 1075-1083.

17 Salahudeen AK, Doshi SM, Pawar T, et al. Incidence rate, clinical correlates, and outcomes of AKI in patients admitted to a comprehensive cancer center. Clin J Am Soc Nephrol 2013; 8: 347-354.

18 Iff S, Craig JC, Turner R, et al. Reduced estimated GFR and cancer mortality. Am J Kidney Dis 2014; 63: 23-30.

19 Chen $\mathrm{C}-\mathrm{Y}$, Lin J-W, Huang J-W, et al. Estimated creatinine clearance rate is associated with the treatment effectiveness and toxicity of pemetrexed as continuation maintenance therapy for advanced nonsquamous non-small-cell lung cancer. Clin Lung Cancer 2015; 16: e131-e140.

20 Chawla LS, Eggers PW, Star RA, et al. Acute kidney injury and chronic kidney disease as interconnected syndromes. $N$ Engl J Med 2014; 371: 58-66.

21 Darmon M, Ciroldi M, Thiery G, et al. Clinical review: specific aspects of acute renal failure in cancer patients. Crit Care 2006; 10: 211.

22 Chauvet $\mathrm{S}$, Courbebaisse $\mathrm{M}$, Ronco $\mathrm{P}$, et al. Pemetrexed-induced acute kidney injury leading to chronic kidney disease. Clin Nephrol 2014; 82: 402-406.

23 Glezerman IG, Pietanza MC, Miller V, et al. Kidney tubular toxicity of maintenance pemetrexed therapy. Am J Kidney Dis 2011; 58: 817-820. 
24 Pujol J-L, Paz-Ares L, de Marinis F, et al. Long-term and low-grade safety results of a phase III study (PARAMOUNT): maintenance pemetrexed plus best supportive care versus placebo plus best supportive care immediately after induction treatment with pemetrexed plus cisplatin for advanced nonsquamous non-small-cell lung cancer. Clin Lung Cancer 2014; 15: 418-425.

25 Calvert AH, Newell DR, Gumbrell LA, et al. Carboplatin dosage: prospective evaluation of a simple formula based on renal function. J Clin Oncol 1989; 7: 1748-1756.

26 Levey AS, Stevens LA, Schmid CH, et al. A new equation to estimate glomerular filtration rate. Ann Intern Med 2009; 150: 604-612.

27 Kidney Disease: Improving Global Outcomes (KDIGO) Acute Kidney Injury Work Group. KDIGO Clinical Practice Guideline for Acute Kidney Injury. Kidney Int 2012; 2: Suppl., 1-138.

28 Kidney Disease: Improving Global Outcomes (KDIGO) CKD Work Group. KDIGO 2012 Clinical Practice Guideline for the Evaluation and Management of Chronic Kidney Disease. Kidney Int 2013; 3: Suppl., 1-150.

29 Perazella MA. Renal vulnerability to drug toxicity. Clin J Am Soc Nephrol 2009; 4: 1275-1283.

30 Sassier M, Dugué AE, Clarisse B, et al. Renal insufficiency is the leading cause of double maintenance (bevacizumab and pemetrexed) discontinuation for toxicity to advanced non-small cell lung cancer in real world setting. Lung Cancer 2015; 89: 161-166.

31 Sato K, Watanabe S, Ohtsubo A, et al. Nephrotoxicity of cisplatin combination chemotherapy in thoracic malignancy patients with CKD risk factors. BMC Cancer 2016; 16: 222.

32 Langer CJ, Paz-Ares LG, Wozniak AJ, et al. Safety analyses of pemetrexed-cisplatin and pemetrexed maintenance therapies in patients with advanced non-squamous NSCLC: retrospective analyses from two phase III studies. Clin Lung Cancer 2017; 18: 489-496. 